\title{
Methacrylate-based polymer blends containing oligomeric 1,2-polybutadienes
}

\author{
Agnieszka Marcinkowska • Ewa Andrzejewska
}

Received: 24 July 2013/ Accepted: 4 October 2013/Published online: 30 October 2013

(c) The Author(s) 2013. This article is published with open access at Springerlink.com

\begin{abstract}
Partly miscible polymer blends with semi-IPNs structure built from polydimethacrylate networks and hydroxyl-terminated liquid polybutadienes with predominant 1,2structure $(\mathrm{LBH})$ were prepared by photopolymerization method. Photopolymerization kinetics of dimethacrylateLBH mixtures were monitored by DSC technique under isothermal conditions. Kinetic curves and related parameters, like polymerization rate and degree of double bond conversion were determined as functions of dimethacrylate structure, LBH molecular mass, and its content in the mixture as well as polymerization temperature. The photopolymerization kinetics and activation energy for the polymerization rate were discussed taking into account the phase separation occurring during the curing reaction. The extent of phase separation (based on $T_{\mathrm{g}}$ 's measurements) depended on $\mathrm{LBH}$ content and dimethacrylate chemical structure. The effect of $\mathrm{LBH}$ content on hardness of polymer blends was also examined.
\end{abstract}

Keywords Polymer blends · Semi-IPN .

1,2-Polybutadienes · Polydimethacrylates

photopolymerization kinetics · DSC

\section{Introduction}

Interpenetrating polymer networks (IPN) are materials comprising two or more polymer networks, which are at least partially interlaced on a molecular scale, but not covalently bonded to each other and cannot be separated unless

A. Marcinkowska $(\varangle) \cdot$ E. Andrzejewska

Institute of Chemical Technology and Engineering, Poznan

University of Technology, 60-965 Poznan, Poland

e-mail: agnieszka.marcinkowska@put.poznan.pl chemical bonds are broken [1]. IPNs are an important class of materials attracting broad interest from both the fundamental and application points of view $[2,3]$. An interesting variation of IPN networks are semi-IPN networks which refers to a class of IPNs where only one of the polymers is crosslinked. On the contrary to IPN network, in semi-IPN systems individual components can be separated from the polymer network components without breaking chemical bonds, so they are generally mixtures of polymers [1]. In most cases polymers are immiscible, or only partially miscible. Immiscible polymers tend to separate spontaneously into two distinct phases. Factors affecting the morphology of the IPNs are compatibility or thermodynamic interaction between components, kinetics of reaction, composition, mobility of the polymer chain, and polymerization degree at the time of gelation [4-7]. It should be noted that the size of domains occurring in a heterogeneous mixture of polymers is related to the rate of polymerization as well as the interactions between polymers.

A group of polymers used as components of IPNs or semi-IPNs are polybutadienes with predominant 1,4structure [8-17]. One of the most common investigations is preparation of IPNs [8-11, 13] or semi-IPNs [12] with methacrylic polymers. In a typical procedure hydroxylterminated butadiene was reacted with isocyanate, and the resulting polymer was swollen with a methacrylate, dimethacrylate, or mixture of methacrylates of various functionality which was then polymerized to form IPN. Some articles describe polymerization of methacrylates in solid 1,4-polybutadiene matrix [14] or grafting of a methacrylate on the polybutadiene during miniemulsion polymerization [15]. High-shock or high-impact polystyrene was obtained by polymerization of styrene in the presence of 1,4-polybutadiene [16] or by mixing of polystyrene with 1,4-polybutadiene [17]. 
An especially interesting group of polybutadienes, not widely investigated so far, are liquid polybutadienes with a high content of 1,2-structure, which are used in the production of rocket fuel [18]. There are only few studies describing mixtures or copolymers of 1,2-polybutadienes with methacrylates [19-21].

Anisimov et al. [19] studied the kinetics of low temperature graft copolymerization of triethylene glycol dimethacrylate (TEGDM) onto an hydroxyl-terminated unsaturated oligomeric butadiene rubber $(\mathrm{MW}=3,200$ ) by infrared spectroscopy. The rate of copolymerization increased with TEGDM concentration, and when the mole fraction of TEGDM in formulation reached 0.96 the reaction rate stopped to increase or even began to decrease which can be associated with the increase in viscosity of the copolymerizing system and diffusion limitations on reacting species. The calculated reactivity ratios were 0.8 and 2.7 for TEGDM and polybutadiene, respectively.

Hydroxyl-terminated but hydrogenated 1,2-polybutadienes were used in preparation of UV-crosslinkable (meth)acrylate modified polyurethanes. These materials had excellent properties what made them useful as flexographic printing plates [21].

In our study we undertook studies on further possible applications of liquid 1,2-polybutadienes using them as components of semi-IPNs in combination with polydimethacrylates. The INPs were prepared in situ by photopolymerization of dimethacrylates containing dissolved polybutadienes. To ensure good miscibility with the monomers (ethylene glycol dimethacrylate, EGDM and 1,6-hexanediol dimethacrylate, HDDM) hydroxyl-terminated polybutadienes (LBH) with MW 2,100, 3,000, and 5,000 were used. The study focuses mainly on the kinetics of photopolymerization taking into account the phase separation occurring during the reaction. The main experimental method used in this study was differential scanning calorimetry (DSC). Isothermal DSC (photo-DSC) is one of the two methods (along with FTIR) most widely used for investigation of photopolymerization kinetics [22, 23]. It reflects very accurately the influence of various factors on the kinetics at any reaction stage (on kinetic curves) and enables measurements at a wide temperature range.

\section{Experimental}

\section{Materials}

Ethylene glycol dimethacrylate (EGDM, Fig. 1) and 1,6hexanediol dimethacrylate (HDDM, Fig. 1) monomers from Aldrich were purified by column chromatography before use. 2,2-Dimethoxy-2-phenylacetophenone (DMPA, $\lambda_{\max } \sim 340 \mathrm{~nm}$ ) photoinitiator from Aldrich was used as received. Three hydroxyl-terminated polybutadienes (Krasol LBH) with a predominance of the 1,2-structure were supplied by Kaučuk Co. (now Sartomer Czech). Krasol LBH 2000 (LBH2, MW 2,100), Krasol LBH 3000 (LBH3, MW 3,000), and Krasol LBH 5000 (LBH5, MW 5,000) are liquid low molecular mass polymers (LBH2 $\mathrm{x} \sim 35$, LBH3 $\mathrm{x} \sim 53$, LBH5 $\mathrm{x} \sim 90$, Fig. 1). They are statistical mixtures of different microstructures: $15 \%$ of cis-1,4-(vinyl) units $25 \%$ of trans-1,4-; and $60 \%$ of 1,2-structural units. They were used without further purification.

\section{Investigated systems}

Combination of two dimethacrylates and three polybutadienes gives six investigated systems. In the frame of each system, ten compositions containing 0-90 mass\% of LBH in 10 mass $\%$ intervals were prepared.

\section{Methods of testing}

Reaction rate profiles and conversions were determined by isothermal DSC (Pyris 6, Perkin Elmer). Throughout the experiments the DSC unit was operated isothermally at the selected temperature kept with accuracy $\pm 0.01{ }^{\circ} \mathrm{C}$. The $5 \mathrm{mg}$ samples were polymerized in open aluminum pans (a diameter $6.6 \mathrm{~mm}$ ). All photopolymerization experiments were conducted at least in triplicate. The polymerization was initiated by the light of a medium pressure Hg lamp (cobalt-glass filter with transmittance range 300-400 nm and maximum at $366 \mathrm{~nm}$, light intensity $2 \mathrm{~mW} \mathrm{~cm}^{-2}$ ) and was carried out in Ar atmosphere. The photoinitiator was used in concentration of 1 mass $\%$. For computations, the heat of polymerization was taken to be $56 \mathrm{~kJ} \mathrm{~mol}^{-1}$ of double bonds [24]. Other details of the experiment were analogous to those described [25].

All the compositions prepared within HDDM/LBH and EGDM/LBH systems and containing the photoinitiator were homogenized in an ultrasonic mixer, in the absence of light to avoid premature polymerization and gave transparent solutions. Viscosities $\eta$ of the investigated compositions were measured by DV-II + PRO Brookfield Rheometer in the cone/plate shear mode.

Thermal transitions (glass temperatures) of semi-IPNs were measured by DSC (Pyris 6, Perkin Elmer). Sample mass of $10-15 \mathrm{mg}$ was scanned at a heating rate of $20{ }^{\circ} \mathrm{C} \mathrm{min}{ }^{-1}$. DSC expression curves were taken by heating the samples in the temperature ranging from -70 to $155^{\circ} \mathrm{C}$.

Samples for hardness testing $(15 \times 10 \times 2.5 \mathrm{~mm})$ were prepared in a steel mould with poly(ethylene terephthalate) (PET) cover to avoid the inhibitory effect of oxygen using a Dymax Light Curing Systems lamp (UV Blue Wave 50). Polymerization was initiated with light of wavelength 
Fig. 1 Chemical structures of monomers: ethylene glycol dimethacrylate (I), 1,6hexanediol dimethacrylate (II), and polybutadienes (III)<smiles>C=C(C)C(=O)OCCOC(=O)C(=C)C</smiles>

(l)<smiles>C=C(C)C(=O)OC(C)(C)CC(C)(C)OC(=O)C(=C)C</smiles>

(II)

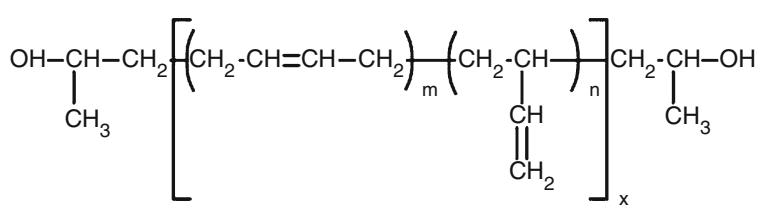

(III)

$366 \mathrm{~nm}$, so PET, which cuts off short-wavelength UV radiation at about $320 \mathrm{~nm}$ did not affect the reaction. Hardness was measured according to standards PN-ISO 868:2004 and PN-EN ISO 527-1:1998 with the use of a Shore (type A) hardness tester (Zwick Roell). The hardness values were the average of at least five measurements.

\section{Results and discussion}

Glass transition and phase separation

During the photocuring process the polymerizing monomer/LBH mixtures undergo phase separation driven by the increase in the molecular mass of the growing dimethacrylate polymer and network formation. In general, the phase separation occurring during the polymerization of a monomer dissolved in a nonreactive component is known as polymerization-induced phase separation.

In the range of 10-50 mass\% of LBH concentrations all the obtained photocured samples are opaque, indicating macrophase separation, which suggests also that the possible copolymerization with polybutadiene double bonds must be rather limited. Above 50 mass $\%$ of $\mathrm{LBH}$ content the samples become optically transparent indicating their homogeneity or a microphase separation with domain size smaller than the visible wavelengths. According to earlier investigations [26, 27], allylic monomers (which include polybutadienes) copolymerize with methyl methacrylate (MMA) to a limited extent, only when the MMA concentration in the feed is high; their polymerization begins when MMA has already reacted to a large extent. At a low MMA content, allylic monomers practically do not copolymerize. Thus, in our investigated systems we can expect two phases: a polydimethacrylate-enriched phase at higher monomer concentrations in the feed and a LBH-enriched phase when the monomer content is low.

The glass temperatures of the materials containing LBH2 are presented in Fig. 2 as a function of the LBH2

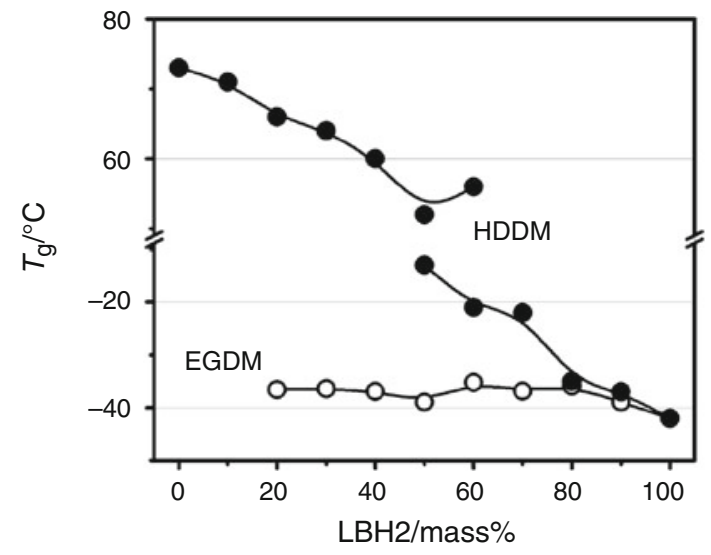

Fig. 2 Glass transition temperatures of semi-IPNs as a function of LBH2 content

concentration. In the case of polyEGDM-based IPNs we observe only one $T_{\mathrm{g}}$ corresponding to $\mathrm{LBH} 2$ phase, which is practically independent of the $\mathrm{LBH}$ content up to about 70 mass $\%$. This $T_{\mathrm{g}}$ is slightly shifted to higher temperatures in comparison to the neat $\mathrm{LBH} 2\left(T_{\mathrm{g}}=-42{ }^{\circ} \mathrm{C}\right)$ indicating incomplete phase separation. The fact that it is very close to the $T_{\mathrm{g}}$ of the neat $\mathrm{LBH} 2$ means that the $\mathrm{LBH} 2$ glass transition is barely influenced by polyEGDM network formation. This suggests in turn that there is no restriction of segmental motion of LBH2's chains by polyEGDM (being in glassy state at $\mathrm{LBH} 2$ glass transition), which corresponds to situation when two polymers form separate domains and degree of mixing is small [5]. At LBH2 content $>80$ mass $\%$ the $T_{\mathrm{g}}$ behavior is dominated by $\mathrm{LBH} 2$ phase. The glass transitions of polyEGDM phase could not be observed in DSC curves what is a general rule in the case of dense polymer networks.

In the case of polyHDDM/LBH2 semi-IPNs the miscibility of the two polymers is much better, which can be concluded from $T_{\mathrm{g}}$ behavior of the semi-IPNs when changing the component ratio. Pure polyHDDM has the glass transition at $73{ }^{\circ} \mathrm{C}$, but its $T_{\mathrm{g}}$ in the semi-IPNs is significantly shifted to lower temperatures with the 


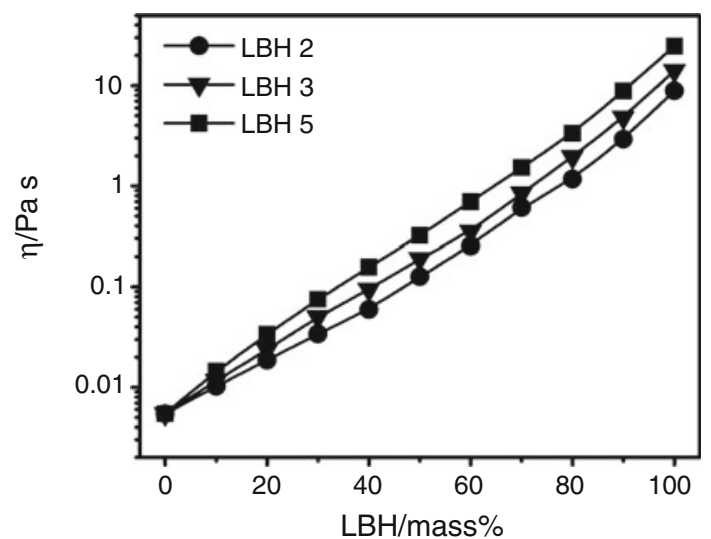

Fig. 3 Viscosity $\eta$ of HDDM/LBH mixtures at $25^{\circ} \mathrm{C}$ as a function of polybutadiene concentration and molecular mass

increasing $\mathrm{LBH} 2$ content. On the other hand, $T_{\mathrm{g}}$ of LBH2 shifts to higher temperatures with increasing polyHDDM content. This indicates on the existence of two phases: polyHDDM-enriched phase containing increasing amounts of LBH2 mixed with polyHDDM at the molecular level and LBH2-enriched phase containing polyHDDM. However, $T_{\mathrm{g}}$ of the latter for compositions containing up to about 50 mass\% of LBH 2 content in the IPN could not be observed due to too small amount of this separated phase. At 50 and 60 mass\% of LBH2 content two $T_{\mathrm{g}}$ values appear showing clearly the existence of the above mentioned two phases. Further increase in LBH2/polyHDDM ratio leads to only one glass transition. This result can be interpreted as (i) the amount of polyHDDM-enriched phase was too small to influence the DSC curve or (ii) no phase separation occurred. The latter situation is possible when the process of separation is stopped by permanent entanglement of the chains [5].

Poorer compatibility of EGDM than HDDM with polybutadienesis also reflected in the values of solubility parameters EGDM 18.2 [28], 18.7 [29], HDDM 18.0 [29, 30], and polybutadiene 17.1 [30].

Photopolymerization kinetics

\section{Viscosity}

The dimethacrylate monomers used in this study have low and similar viscosities in $25^{\circ} \mathrm{C} \eta$ of HDDM is $0.0055 \mathrm{~Pa} \mathrm{~s}$ and of EGDM $0.003 \mathrm{~Pa}$ s. On the other hand, the viscosities of liquid polybutadienes are much higher in $25^{\circ} \mathrm{C}$ they are $8.87,14.09$, and $24.76 \mathrm{~Pa} \mathrm{~s}$ for $\mathrm{LBH} 2, \mathrm{LBH} 3$, and LBH5, respectively. Thus, the viscosities of monomer/ polybutadiene mixtures increase significantly with the LBH content as shown in Fig. 3. The range of viscosities of the investigated formulations is very wide and covers four
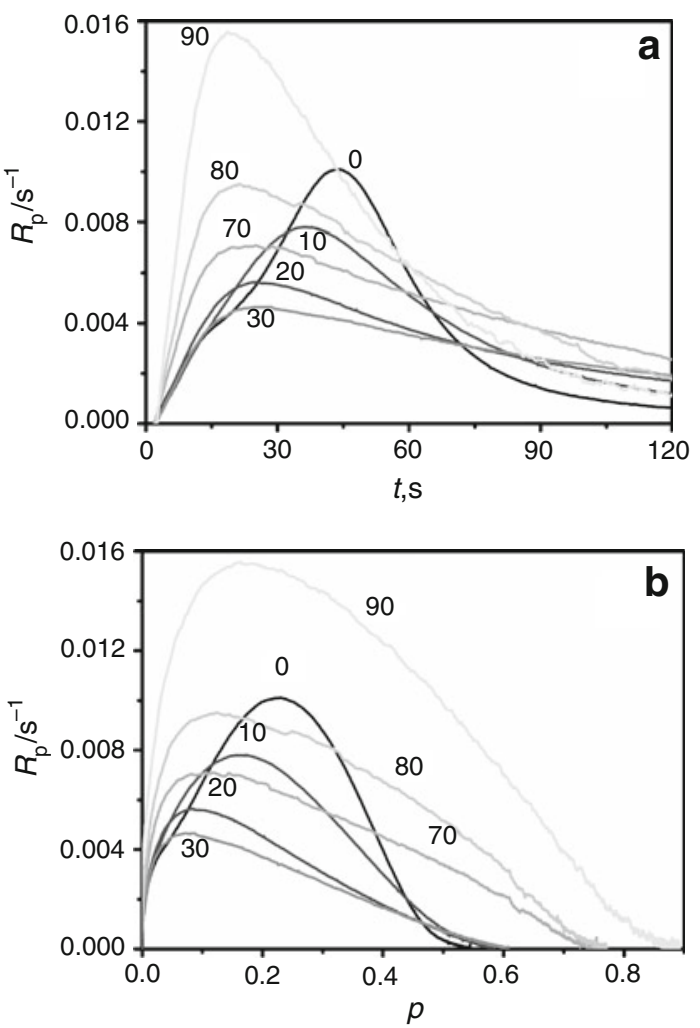

Fig. 4 Rate of polymerization $R_{\mathrm{p}}$ as a function of $\mathbf{a}$ irradiation time $t$, b double bond conversion $p$ for HDDM/LBH3 system; the numbers indicate the LBH3 content (mass\%) in the feed; polymerization temperature $25^{\circ} \mathrm{C}$

orders of magnitude $\left(10^{-3}-10 \mathrm{~Pa}\right.$ s), which, as will be shown, influences the photocuring kinetics.

\section{Influence of polybutadiene molecular mass and concentration}

The polymerization rates and conversions presented in Figs. 4, 5, 6, 7, 8, and 9 are referred to methacrylic double bonds without considering the double bonds from LBH. As mentioned above, the copolymerization of allylic monomers with methacrylates is limited. Although there are reports that polybutadienes may somewhat copolymerize with methacrylates $[1,31]$ this copolymerization is not taken into account in kinetic analysis [31]. Moreover, under our experimental conditions the LBH homopolymerization does not occur (no reaction heat is observed during photo-DSC measurements). However, we cannot completely exclude the copolymerization, especially at low LBH concentration [26, 27], and for this reason our results indicate trends rather than the actual parameters of the reaction.

Figure 4 presents the exemplary dependences of the polymerization rate $\left(R_{\mathrm{p}}\right)$ on irradiation time $(t)$ and double 


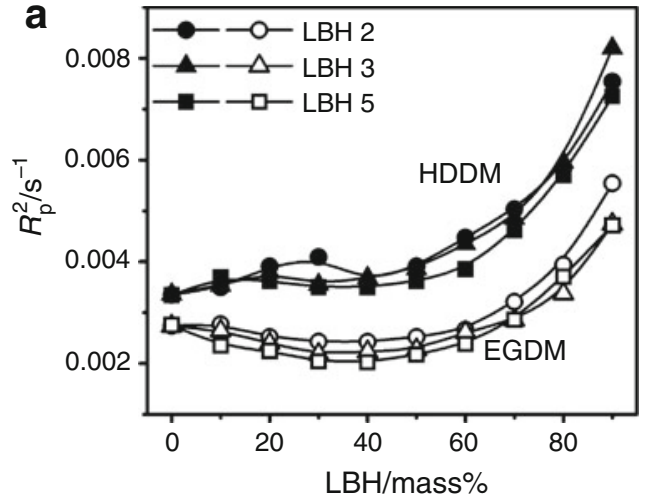

b

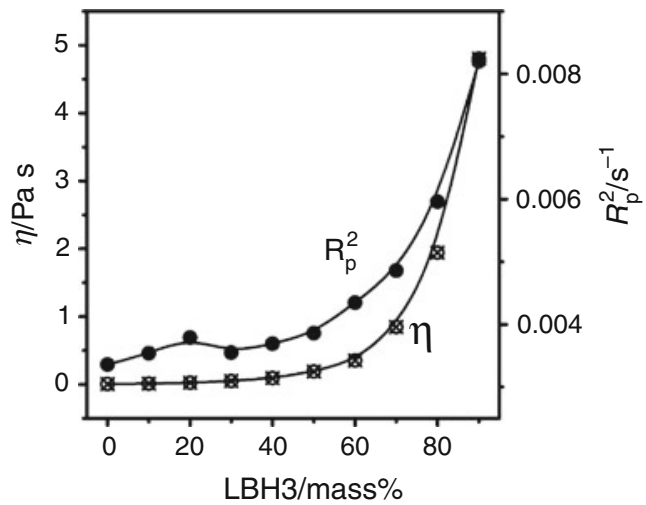

Fig. 5 a Rate of polymerization at $2 \%$ of double bond conversion $R_{\mathrm{p}}^{(2)}$ versus LBH content for HDDM/LBH and EGDM/LBH systems b initial viscosity and $R_{\mathrm{p}}^{(2)}$ of HDDM/LBH3 system versus $\mathrm{LBH} 3$ content; polymerization temperature $25{ }^{\circ} \mathrm{C}$

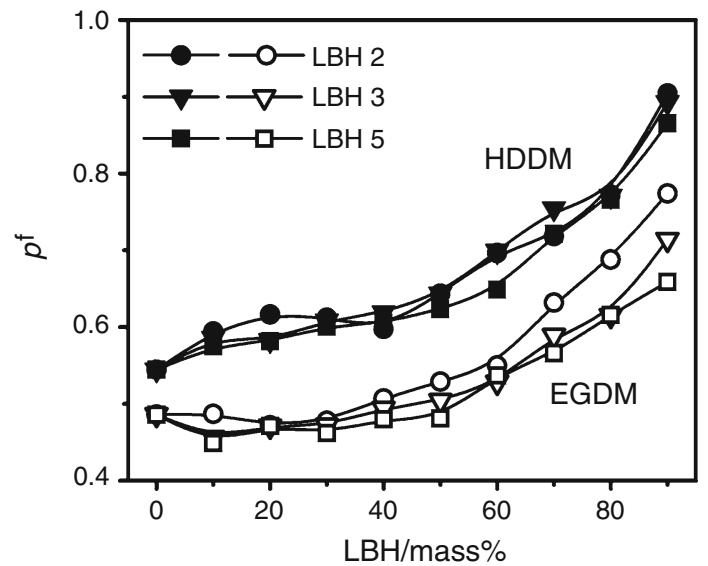

Fig. 6 Final double bond conversion, $p^{\mathrm{f}}$ as a function of polybutadiene content and polybutadiene molecular mass for HDDM/LBH and EGDM/LBH systems

bond conversion $(p)$ for the photopolymerization of the HDDM/LBH3 mixtures at $25{ }^{\circ} \mathrm{C}$. Kinetic curves obtained for other dimethacrylate/LBH systems behave similarly. The DSC traces registered for the neat HDDM have a bimodal shape typical for the polymerization of a

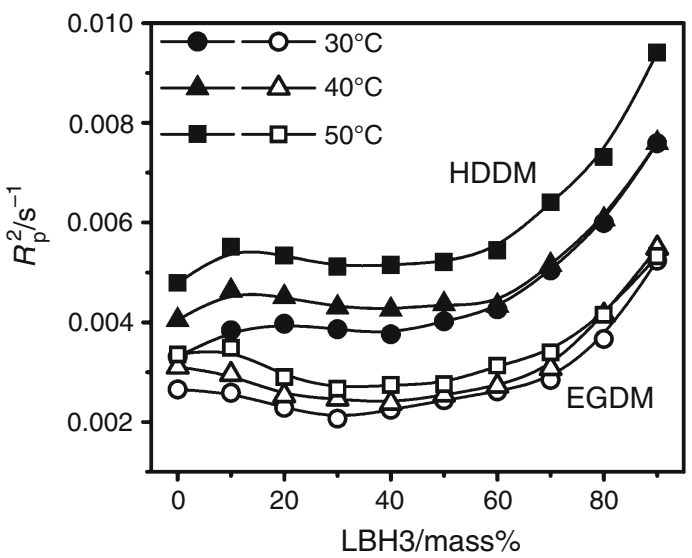

Fig. 7 Rate of polymerization at $2 \%$ of double bond conversion $R_{\mathrm{p}}^{(2)}$ versus LBH content at three different polymerization temperatures

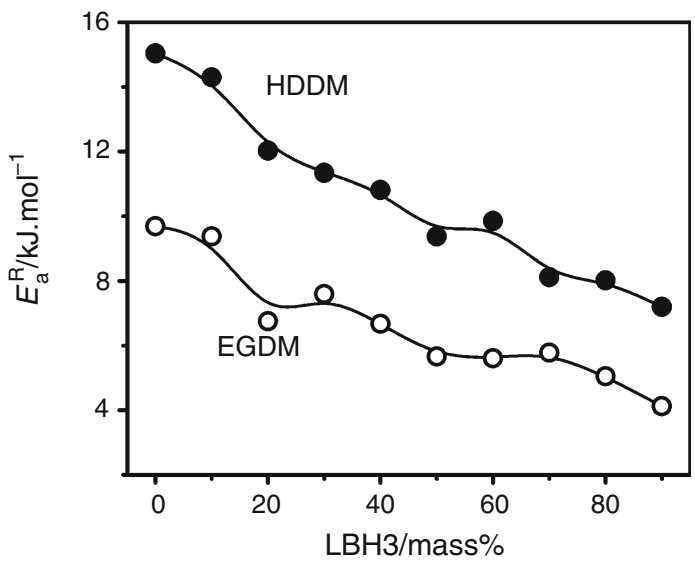

Fig. 8 Activation energy for the polymerization rate at $2 \%$ of double bond conversion

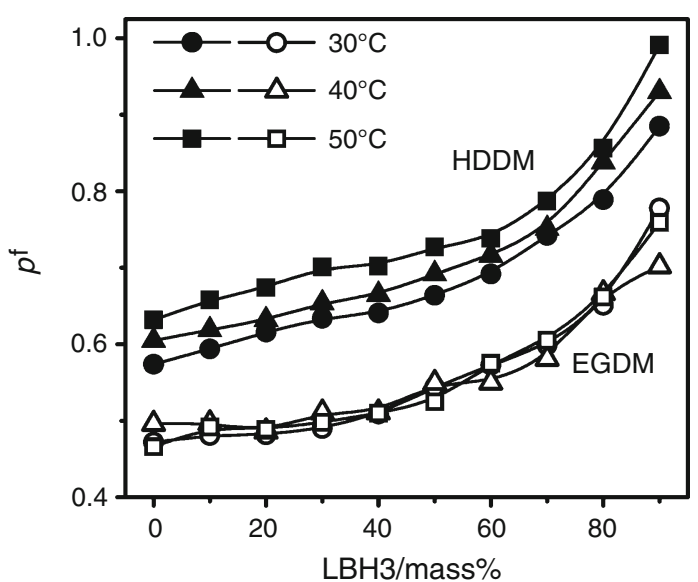

Fig. 9 Final double bond conversion $p^{\mathrm{f}}$ as a function of LBH3 content and polymerization temperature for HDDM/LBH and EGDM/ LBH systems

multifunctional monomer of low viscosity [14]. The $R_{\mathrm{p}}=f(p)$ curve shows a slight inflection point at about 4-5\% of double bond conversion, which corresponds to 
the beginning of autoacceleration. At about $23 \%$ of double bond conversion the polymerization rate reaches its maximum value, $R_{\mathrm{p}}^{\max }$ associated with the end of the gel effect.

As LBH3 is added $R_{\mathrm{p}}^{\max }$ decreases and when the polybutadiene content reaches 30 mass $\%$, the autoacceleration is supressed due to the increase in mobility of macroradicals as a result of dilution and plasticisation of the system; the efficiency of radical termination becomes nearly the same throughout the entire polymerization [32]. On the other hand, the rate of polymerization on the shoulder of the curve, prior to autoacceleration, increases with increasing concentrations of LBH. This is a consequence of the increased initial viscosity of the system and resulting enhanced diffusional limitations of the termination process in classical kinetics the termination rate coefficient $k_{\mathrm{t}}^{\mathrm{b}}$ is inversely proportional to the viscosity $\left(k_{\mathrm{t}}^{\mathrm{b}} \sim 1 / \eta\right)$, and the polymerization rate $R_{\mathrm{p}}$ is related to the termination rate coefficient as $R_{\mathrm{p}} \sim 1 /\left(k_{\mathrm{t}}^{\mathrm{b}}\right)^{0.5}$. The rates of polymerization at $2 \%$ of double bond conversion $\left(R_{\mathrm{p}}^{(2)}\right)$ versus LBH content are shown in Fig. $5 \mathrm{a}$.

In the case of HDDM/LBH systems, a slight increase of the polymerization rate $R_{\mathrm{p}}^{(2)}$ is observed up to about 50 mass\% of LBH content. Further, increase in LBH concentration results in a rapid acceleration of the polymerization. This behavior correlates qualitatively well with changes of initial viscosity during increasing LBH content (Fig. 5b). However, a quantitative correlation of these parameters cannot be found due to the influence of additional parameters on the polymerization rate. In general, the polymerization rates of EGDM/LBH systems are lower than these of HDDM/LBH systems due to the formation of denser polymethacrylate networks [33]. In this case, even a slight decrease in $R_{\mathrm{p}}^{(2)}$ is observed before the rapid growth of the rate at higher LBH contents. This may point to a retardation of the polymerization, which is better observable in the concentration range where the increase in viscosity is not large (LBH content $0-50$ mass\%). The retardation can result from a partial copolymerization with LBH in nonseparated domains and extensive chain transfer with the formation of allyl-type radicals of very low reactivity (degradative chain transfer) [22]. The chain transfer from the propagating methacrylate radicals to polybutadiene is less probable due to lowered reactivity of these radicals toward chain transfer and enhanced reactivity toward propagation [22].

However, very high initial viscosity of the investigated systems affects also the propagation rate, which can be concluded from the reduction of the polymerization rate when LBH molecular mass is increased. In our case, such reduction is not high due to rather small differences between viscosities of the investigated systems. Under such conditions diffusional limitations on propagation begin to occur at increasingly smaller conversions; this leads to a reduction of the polymerization rate. The observable influence of viscosity on $R_{\mathrm{p}}^{(2)}$ points to the lack of phase separation at low conversions. Separation appears when the polymethacrylate chains reach a suitable length. The viscosity effects are less pronounced in EGDM/LBH systems due to poorer compatibility between the polyEGDM network and LBH.

As long as the visible phase separation is observed, the final double bond conversion $p^{\mathrm{f}}$ practically does not depend on LBH contents (for polyEGDM) or depends only slightly (for polyHDDM), as can be seen from Fig. 6. The lack of this dependence observed in the first case suggests that the EGDM polymerization occurs in the separated phase (despite the initial full miscibility of the components). When polymerized material becomes visually homogeneous, $p^{\mathrm{f}}$ starts to increase with LBH content indicating in turn that the polydimethacrylate network swollen by polybutadiene has greater mobility, which enables higher conversion of double bonds. Better compatibility of polyHDDM with LBH causes that $p^{\mathrm{f}}$ slightly increases even in the presence of small amounts of LBH, and the phase separation is partial. These results are supported by $T_{\mathrm{g}}$ behavior. However, as mentioned above, we cannot completely exclude LBH (co)polymerization.

\section{Effect of temperature}

The polymerization kinetics was followed at 25, 30, 40, and $50{ }^{\circ} \mathrm{C}$. The $R_{\mathrm{p}}^{(2)}$ values registered at 30,40 , and $50{ }^{\circ} \mathrm{C}$ are shown in Fig. 7 as a function of LBH3 content.

As could be expected, the polymerization rate increases with temperature, but stronger for HDDM-based systems. This indicates that the activation energy for the polymerization rate $E_{\mathrm{a}}^{\mathrm{R}}$ is higher for these systems, which is illustrated in Fig. 8 showing this parameter calculated for the reaction rate at $2 \%$ of conversion.

In the case of photochemical initiation, $E_{\mathrm{a}}^{\mathrm{R}}$ is expressed by the following equation:

$E_{\mathrm{a}}^{\mathrm{R}}=E_{\mathrm{a}}^{\mathrm{p}}-0.5 \cdot E_{\mathrm{a}}^{\mathrm{t}}$,

where $E_{\mathrm{a}}^{\mathrm{p}}$ denotes the activation energy for propagation, and $E_{\mathrm{a}}^{\mathrm{t}}$ is the activation energy for termination (activation energy of initiation is close to zero). Lower $E_{\mathrm{a}}^{\mathrm{R}}$ values for EGDM-based systems can result from higher $E_{\mathrm{a}}^{\mathrm{t}}$ of EGDM polymerization due to formation of denser polymer network hindering movements of radical ends. On the other hand, activation energy for termination corresponds to temperature dependence of inverse viscosity of the system [34]. Such dependence is usually high for viscous monomers and resins. Thus, the decrease of $E_{\mathrm{a}}^{\mathrm{R}}$ with increasing LBH content results mainly from an increase in $E_{\mathrm{a}}^{\mathrm{t}}$ in Eq. (1). 


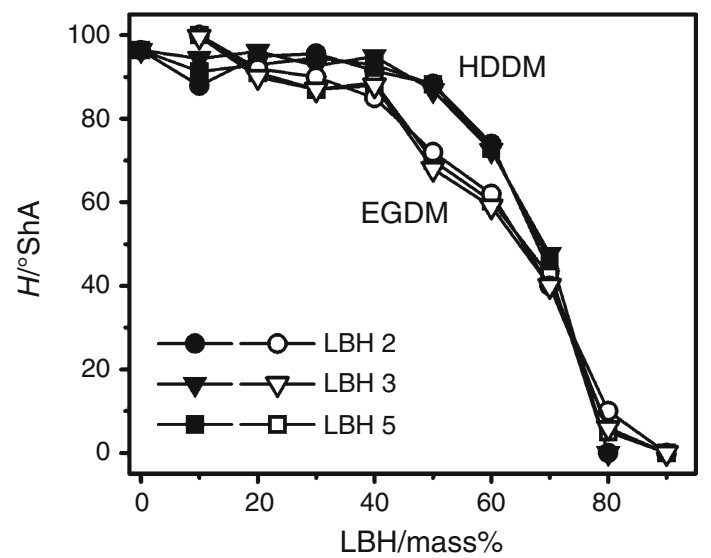

Fig. 10 Shore A hardness $H$ as a function of polybutadiene content

The increase in the polymerization temperature results in enhanced phase separation. Instability of the system is driven by the progressive increase of the average molecular mass of the polymerizing species; the degree of demixing depends on the competition between phase separation dynamics and reaction kinetics. Due to rapid decrease of viscosity with increasing temperature, diffusion of polymer chains from each other becomes easier, which facilitates the phase separation.

The increase in polymerization temperature causes also an increase in the final conversion degree (Fig. 9). At higher temperatures the mobility of reacting species increases allowing the system to react for a longer time and reach higher conversions. In the case of EGDM-containing systems the temperature affects the conversion only slightly, which can be related, i.e., to the slight effect of temperature on the polymerization rate.

\section{Hardness testing}

The obtained semi-IPN materials were tested for their Shore A hardness. The results are shown in Fig. 10. Interestingly, the hardness remains practically constant for HDDM-based systems up to LBH content 40 mass\% and rapidly drops at higher LBH addition. For the EGDM-based systems the hardness decreases slightly with increasing LBH content and similarly as in the previous case, rapidly decreases when LBH content exceeds 40 mass\%. Generally, it is lower for polyHDDM-containing semi-IPNs. It is against expectation that EGDM-based materials will be harder due to more dense methacrylate network. The observed higher hardness of materials with polyHDDM matrix can result from higher double bond conversion leading to higher crosslink density (Fig. 8).

The observed behavior of the hardness with increasing LBH content can be explained by phase separation observed in the materials containing up to about 50 mass $\%$ of the linear polymer. Such a physical structure results in toughening of the glassy polymer matrix, because the LBH dispersion facilitates shear yielding of the matrix and hence cracktip blunting [12]. This may be the reason of the practically constant blend hardness to the LBH content of about 30-50 mass\% (especially in the case of HDDM/LBH systems). Better compatibility of the polymeric components in this semi-IPN results in better adhesion at their interphase which positively influences mechanical properties. Above this LBH level the two polymer phases become compatible, and $\mathrm{LBH}$ begin to act as a plasticizer causing stepwise decrease of hardness.

\section{Conclusions}

Partly miscible polymer blends of polyHDDM and polyEGDM with LBHs of various lengths were obtained in the form of semi-IPNs. In the case of polyHDDM-containing semi-IPNs the miscibility of the two polymers is much better, which can be concluded from semi-IPN's $T_{\mathrm{g}}$ behavior. The extent of phase separation depends on LBH content; materials containing LBH in amounts exceeding 50 mass\% become optically homogeneous.

Addition of LBH to the monomers results in suppression of the gel effect; the polymerization rate at low conversions does not depend on LBH content up to about 50 mass\%, but rapidly increases at higher contents, which is associated with the disappearance of the phase separation and the resulting increase in the viscosity of the polymerizing medium.

The phase separation affects hardness of the materials. The hardness remains practically constant (polyHDDM matrix) or changes only slightly (polyEGDM matrix) when the phase separation takes place, but when the blends become homogeneous the hardness rapidly decreases due to plasticization of the material by $\mathrm{LBH}$.

Acknowledgements This study was supported by the Research Project of Poznan University of Technology: 32-379/2013 DS-PB. The authors would like to acknowledge the support from MSc Katarzyna Wegner for help in the experimental study.

Open Access This article is distributed under the terms of the Creative Commons Attribution License which permits any use, distribution, and reproduction in any medium, provided the original author(s) and the source are credited.

\section{References}

1. Wilkinson A, McNaught AD. IUPAC compendium of chemical terminology. 2nd ed. Cambridge: Royal Society of Chemistry; 1997.

2. Bischoff R, Cray SE. Polysiloxanes in macromolecular architecture. Prog Polym Sci. 1999;24:185-219. 
3. Shivashankar M, Mandal BK. A review on interpenetrating polymer network. Int J Pharm Pharm Sci. 2013;4:1-7.

4. Chen F, Cook WD. Curing kinetics and morphology of NEGDMA/DGEBA IPNs via sequential photo and thermal polymerization. Eur Polym J. 2008;44:1796-813.

5. Karabanova LV, Boiteux G, Seytre G, Stevenson I, Lloyd AW, Mikhalovsky SV, Helias M, Sergeeva LM, Lutsyk ED, Svyatyna A. Phase separation in the polyurethane/poly(2-hydroxyethyl methacrylate) semi-interpenetrating polymer networks synthesized by different ways. Polym Eng Sci. 2008;48:588-97.

6. Duran H, Meng S, Kim N, Hu J, Kyu T, Natarajan LV, Tondiglia VP, Bunning TJ. Kinetics of photopolymerization-induced phase separation and morphology development in mixtures of a nematic liquid crystal and multifunctional acrylate. Polymer. 2008;49:534-45.

7. Lipatov YS, Alekseeva TT. Phase-separated interpenetrating polymer networks. Berlin: Springer-Verlag GmbH; 2007.

8. Vidal F, Juger J, Chevrot C, Teyssié D. Interpenetrating polymer networks from polymeric imidazolium-type ionic liquid and polybutadiene. Polym Bull. 2006;57:473-80.

9. Shaplov AS, Goujon L, Vidal F, Lozinskaya EI, Meyer F, Malyshkina IA, Chevrot C, Teyssie D, Odinets IL, Vygodskii YS. Ionic IPNs as novel candidates for highly conductive solid polymer electrolytes. J Polym Sci Part A. 2009;47:4245-66.

10. Plesse C, Vidal F, Gauthier C, Pelletier JM, Chevrot C. Teyssie. Poly(ethylene oxide)/polybutadiene based IPNs synthesis and characterization. Polymer. 2007;48:696-703.

11. Tsai M-H, Huang S-L, Chang P-H, Chen C-J. Properties and pervaporation separation of hydroxyl-terminated polybutadienebased polyurethane/poly(methyl metharcylate) interpenetrating networks membranes. J Appl Polym Sci. 2007;106:4277-86.

12. Vidal F, Plesse C, Teyssié D, Chevrot C. Long-life air working conducting semi-IPN/ionic liquid based actuator. Synth Met. 2004;142:287-91.

13. Pham S, Burchill PJ. Toughening of vinyl ester resins with modified polybutadienes. Polymer. 1995;36:3279-85.

14. Mateo JL, Calvo M, Bosch P. Photoinitiated polymerization of methacrylic monomers in a polybutadiene matrix $(\mathrm{PB})$ : kinetic, mechanistic, and structural aspects. J Polym Sci Part A. 2001;39:2444-53.

15. Pham TTB, Fellows CM, Gilbert RG. Grafting of dodecyl methacrylate onto hydroxylated polybutadiene by miniemulsion polymerization. J Polym Sci Part A. 2004;42:3404-16.

16. Coutinho FMB, Costa MPM, Guimaraes MJOC, Soares BG. Preparation and characterization of high-impact polystyrene using different types of polybutadiene. J Appl Polym Sci. 2008;108:406-13.

17. Soto G, Nava E, Rosas M, Fuenmayor M, Gonzalez IM, Meira GR, Oliva HM. Bulk polymerization of styrene in the presence of polybutadiene: effect of initiator type and prepolymerization conditions on particle morphology. J Appl Polym Sci. 2004;92:1397-412.

18. Panicker SS, Ninan KN. Influence of molecular weight on the thermal decomposition of hydroxyl terminated polybutadiene. Thermochim Acta. 1997;290:191-7.
19. Anisimov Yu A, Nikolova ON, Anisimov Yu N. Low-temperature graft copolymerization of triethylene glycol dimethacrylatewith oligomeric butadiene Rubber. Russ J Appl Chem. 2011;84:902-5.

20. Liaw D-J, Lin L-L. Studies on graft copolymerization of cyclohexyl methacrylate onto 1,2-polybutadiene. J Appl Polym Sci. 1989;37:1993-2006.

21. Gensho T, Masamitsu Y, Masakazu K, Yoneharu T, Kazuhiro S. Photosensitive compositions containing hydrogenated 1,2-polybutadiene. Patent 4192684, 11 Mar 1980.

22. Esen DS, Karasu F, Arsu N. The investigation of photoinitiated polymerization of multifunctional acrylates with TX-BT by Photo-DSC and RT-FTIR. Prog Org Coat. 2011;70:102-7.

23. De Brito M, Courtecuisse F, Allonas X, Croutxe-Barghorn C. Evaluation of the exothermicity of free radical photopolymerization through a RT-FTIR and PhotoDSC study. J Photopolym Sci Technol. 2010;6:765-9.

24. Odian G. Principles of polymerization. 4th ed. Hoboken: Wiley; 2004.

25. Andrzejewska E, Socha E, Andrzejewski M. Cross-linking photocopolymerization of dodecyl methacrylate with oxyethylene glycol dimethacrylates: kinetics and reactivity ratios. Polymer. 2006;47:6513-23.

26. Hendrana S, Hill DJT, Perera MCS, Pomery PJ. Copolymerization of methyl methacrylate and allyl acetate. Part I. Rate of reaction. Polym Int. 2001;50:597-605.

27. Hill DJT, Perera MCS, Pomery PJ, Joseph E. Copolymerization of methyl methacrylate and diethylene glycol bis(allyl carbonate). Polymer. 1997;38:695-702.

28. Okay O. Prog Polym Sci. 2000;25:711-79.

29. Xu Z, Yang L, Wang Q. Different alkyl dimethacrylate mediated stearyl methacrylate monoliths for improving separation efficiency of typical alkylbenzenes and proteins. J Chromatogr A. 2009;1216:3098-106.

30. Mateo JL, Calvo M, Bosch P. Photoinitiated polymerization of methacrylic monomers in a poly(methyl methacrylate) matrix: a comparative study with other matrices (styrene-butadiene-styrene, polystyrene, and polybutadiene). J Polym Sci Part A. 2002;40:120-7.

31. Dickens SH, Stansbury JW, Choi KM, Floyd CJE. Macromolecules. 2003;36:6043-53.

32. Young JS, Bowman CN. Effect of polymerization temperature and cross-linker concentration on reaction diffusion controlled termination. Macromolecules. 1999;32:6073-81.

33. Elliott JE, Lovell LG, Bowman CN. Primary cyclization in the polymerization of bis-GMA and TEGDMA: a modeling approach to understanding the cure of dental resins. Dent Mater. 2001;17:221-9.

34. Barner-Kowollik C, Vana P, Davis TP. The kinetics of free radical polymerization. In: Matyjaszewski K, Davis TP, editors. Handbook of radical polymerization. Hoboken: Wiley; 2002. p. $187-263$. 